\section{A rare case of spinal dural arteriovenous fistula}

\author{
Mariya Apostolova, 1 Samer Nasser, ${ }^{1}$ \\ Samir Kodsi2 \\ 1Department of Internal Medicine; \\ 2Department of Radiology, Conemaugh \\ Memorial Medical Center, Johnstown, PA, \\ USA
}

\begin{abstract}
Spinal dural arteriovenous fistula (SDAVF) is a rare vascular malformation of the spine. Only a limited number of cases of SDAVF have been reported in the current literature. We describe the case of a 74 year old male who presented with gradually progressive bilateral lower extremity weakness and bladder dysfunction and was subsequently diagnosed with SDAVF affecting both the thoracic and lumbar spine. The patient later underwent embolization with some improvement in his neurologic symptoms.
\end{abstract}

\section{Case Report}

A 74 year old male with a past medical history significant for hypertension, hypercholesterolemia, hypothyroidism, and Barrett's esophagus presented with gradually progressive bilateral lower extremity weakness and urinary retention. The symptoms started a month prior to presentation. The patient gradually developed difficulty with ambulation to the point that he needed assistance getting up and with transfers. Upon admission chest X-ray showed cardiomegaly without evidence of acute infiltrates or effusions. Sedimentation rate was $8 \mathrm{~mm} / \mathrm{hr}$. Lyme disease antigen was negative. Electrolytes were within normal limits: sodium $145 \mathrm{mE} / \mathrm{L}$, potassium $3.5 \mathrm{mEq} / \mathrm{L}$, chloride $107 \mathrm{mEq} / \mathrm{L}$, BUN $28 \mathrm{mg} / \mathrm{dL}$, Cr $0.9 \mathrm{mg} / \mathrm{dL}$, glucose $102 \mathrm{mg} / \mathrm{dL}$, calcium $9.6 \mathrm{mg} / \mathrm{dL}$. CBC showed WBC 4.6 thou/cumm, hemoglobin $13.4 \mathrm{gm} / \mathrm{dL}$, hematocrit $39 \%$, MCV 96 FL, platelet count 120 thou/cumm, polys $64 \%$. Urinalysis was negative. Folate level was > $20 \mathrm{ng} / \mathrm{mL}$, vitamin B12 level was 667 $\mathrm{pg} / \mathrm{ml}$, HTLV antibody was negative, RPR was nonreactive. ANA was negative and TSH level was $2.4 \mathrm{uIU} / \mathrm{mL}$.

Unenhanced magnetic resonance imaging (MRI) of the cervical spine showed no evidence of cord signal abnormality or evidence of fracture. Unenhanced MRI of the thoracic spine showed abnormal hyperintense T2 signal and hypointense T1 signal extending from the $\mathrm{T} 6$ vertebral body level down to the conus medullaris with smooth mild cord expansion at the conus medullaris (Figure 1). A flame shaped hyperintense T2 signal abnormality sparing the chord peripherally with a low peripheral T2 signal throughout the intradural central canal. The findings were compatible with Type I dural arteriovenous fistula. Combined, pre- and post-contrast MRI of the thoracic spine revealed enhancement of all the serpiginous intradural blood vessels along the posterior aspects of the thoracic spinal cord (Figure 2). This strengthened the leading diagnosis of Type I spinal dural arteriovenous fistula (SDAVF). There was no evidence of intramedullary enhancement. There were multiple flow voids noted on the T2 sequence within the intradural space and surrounding the cauda equina nerve roots. Follow-up post-contrast study of the lumbar spine showed evidence of enhancing vessels in an intradural location, surrounding the conus medullaris (Figures 3 and 4). Additional MRI images are illustrated in Figures 5-9.

\section{Discussion}

SDAVF is a rare spinal vascular malformation which can lead to significant morbidity when left untreated. A number of classifications that reflects the complex anatomy of these vascular malformations have been proposed.1,2 The arteries that supply the spinal cord eventually branch off into meningeal arteries. ${ }^{2}$ The point at which a meningeal artery pierces the dura represents a potential site for the development of dural arteriovenous fistula. ${ }^{2}$ Based on a review of the records of 81 patients with spinal arteriovenous lesions, Rosenbaum et al. classified spinal arteriovenous lesions into four types, the most common of which is the SDAVF, Type I.3,4 Depending on the number of arterial feeders, Type I spinal arteriovenous fistulas are divided into Type Ia and Type Ib. ${ }^{3-5}$ A Type Ia fistula has one feeder and a Type Ib fistula has multiple feeders. ${ }^{3-5}$ The nidus of a SDAVF is located in the dura covering the proximal nerve root and adjacent spinal dura.3,4 Additionally, intradural arteriovenous malformations (AVMs) are classified into glomus (Type II), juvenile (Type III), and direct (Type IV). ${ }^{3,4}$ In juvenile AVMs, the vascular nidus lies completely within the cord parenchyma. ${ }^{4}$ In glomus AVMs, the vascular nidus lies partially within the cord parenchyma. ${ }^{4}$ In direct AVMs, there is no intervening glomus of vessels. ${ }^{4}$ In a retrospective analysis of 156 SDAVF cases, $67.6 \%$ were found in the thoracic region and $18.6 \%$ in the lumbar region. ${ }^{6}$ Cases of multiple SDAVFs in a single patient have rarely been reported. $., 6,7$

The pathophysiology of SDAVFs is unclear but they are thought to be acquired.4-6 The lesion is more common in men older than 50 years
Correspondence: Samir Kodsi, Conemaugh Memorial Medical Center, Department of Radiology, 1086 Franklin Street, Johnstown PA 15905, USA.

E-mail: kodsis@me.com

Key words: arteriovenous fistula, spinal dural arteriovenous fistula, spinal arteriovenous fistula, SDAVF

Acknowledgements: we thank Dr. Kotadia and D. Livingston for critical review of this manuscript.

Conflict of interests: the authors report no conflict of interests.

Contributions: MA, SN, SK, were involved in data gathering, drafting the manuscript and revising it critically.

Received for publication: 12 August 2012. Accepted for publication: 10 September 2012

This work is licensed under a Creative Commons Attribution NonCommercial 3.0 License (CC BYNC 3.0).

(C) Copyright M. Apostolova et al., 2012

Licensee PAGEPress, Italy

Neurology International 2012; 4:e19

doi:10.4081/ni.2012.e19

old. 5,6 It usually affects the thoracic and lumbar spine.1,5-7 These patients often present with progressive myelopathy, claudication, sensory loss, bowel and bladder dysfunction. In SDAVFs, a dural branch of a segmental spinal artery fills the pial coronal venous plexus by draining into a medullary vein. This leads to ischemia and edema which in turn causes progressive myelopathy. The median time from symptom onset to diagnosis falls in the range of 12-44 months.?

A SDAVF appears as a hyperintense lesion on T2-weighted images, and often a corresponding hypointense signal can be found on T1-weighted images. ${ }^{1,3,5-8}$ The findings on the T-2 weighted images are secondary to cord ischemia and edema. Additional MRI findings include prominent intradural veins, spinal cord enhancement, enlargement, scalloping and irregular cord surface. ${ }^{3,5}$ Additionally, MRI will be insufficient in the evaluation for multiple SDAVFs. Although the above MRI findings are nonspecific, they should prompt further investigation with arteriography. ${ }^{3}$ Some authors still advocate the use of spine myelography before angiography. 5 Myelography is recommended by some authors for patients whose clinical findings are consistent with SDAVF but have negative or equivocal MRI findings. ${ }^{3,5}$ Postgadolinium MR angiography can aid in the diagnosis of SDAVFs by supplementing the information provided by the MRI. 5 It can depict abnormal intradural vessels, 


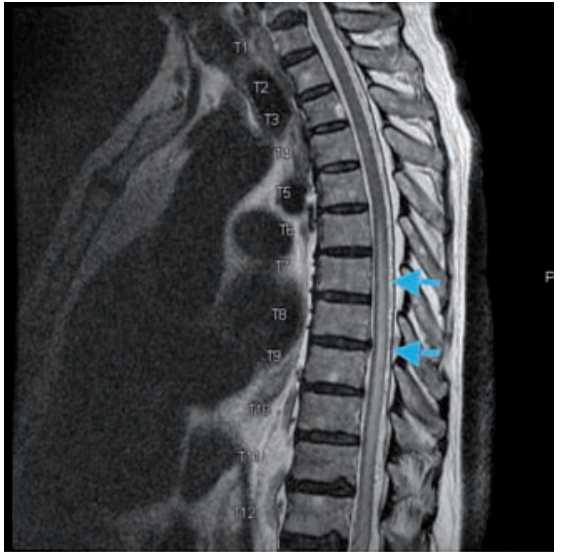

Figure 1. Sagittal T2-weighted image of the thoracic spine demonstrate hyper intense T2 signal abnormality within the central area of the thoracic spinal cord extending from T6 to the conus medullaris. Multiple intradural flow voids are noted (arrows).

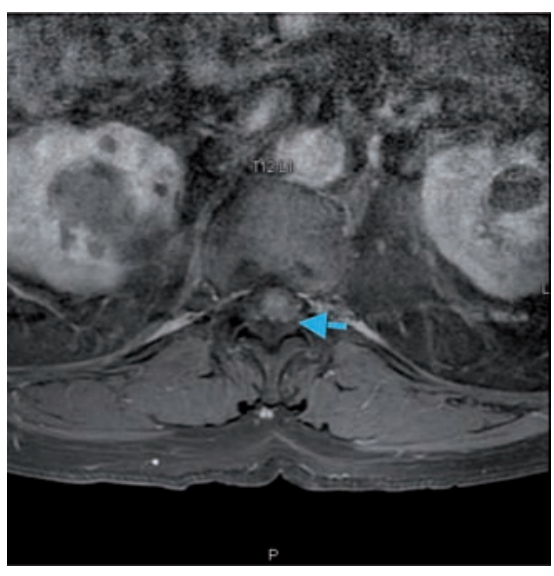

Figure 4. Axial T1 FAT saturated post contrast image shows enhancement of prominent intradural veins (arrow).

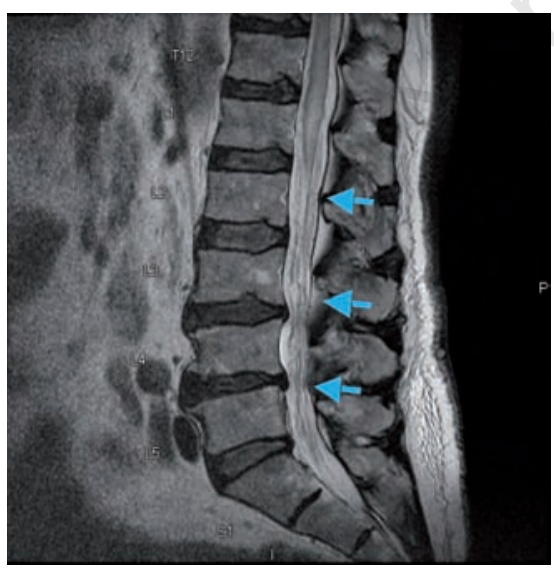

Figure 7. Sagittal T2-weighted sequence shows hyper intense abnormality throughout the conus medullaris with sparing of the periphery. Multiple intradural flow voids are seen from the level of the conus medullaris down to the cauda equina nerve roots (arrows).

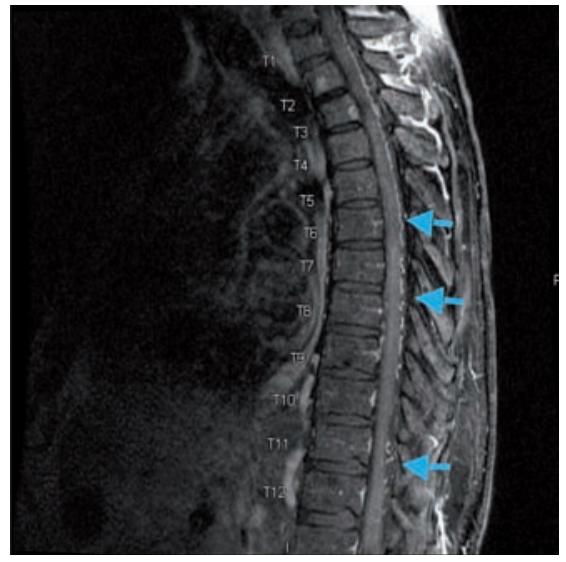

Figure 2. Post-contrast image of the thoracic spine demonstrates multiple

intradural flow voids (arrows).

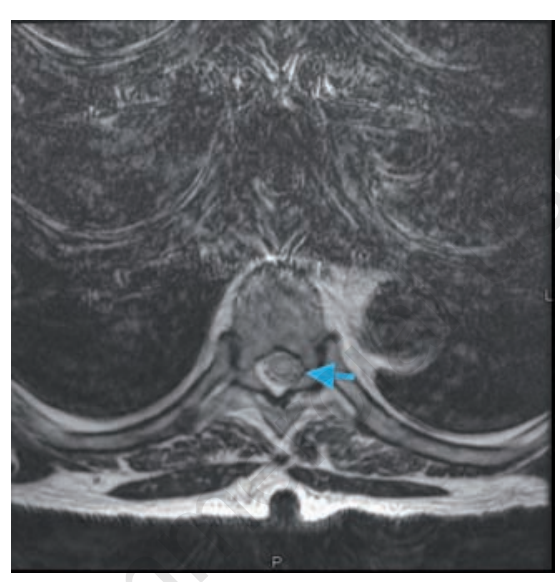

Figure 5. Axial T2 sequence shows hyper intense T2 signal in the central aspect of the thoracic spinal cord with several prominent intradural flow voids (arrow).

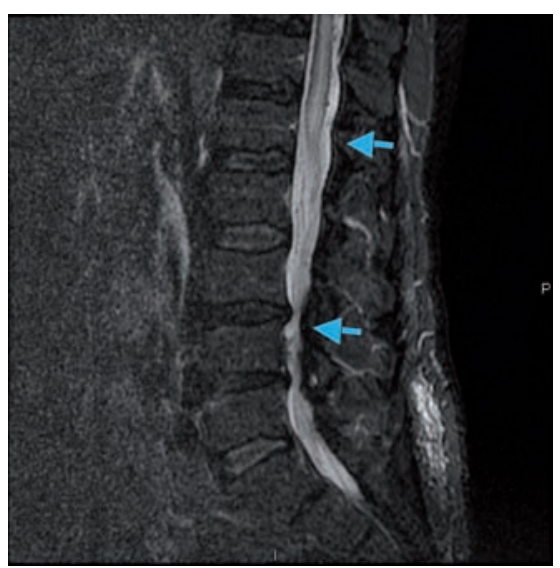

Figure 8. STIR sequence of the lumbar spine shows abnormality throughout the conus medularis with sparing of the periphery (arrows).

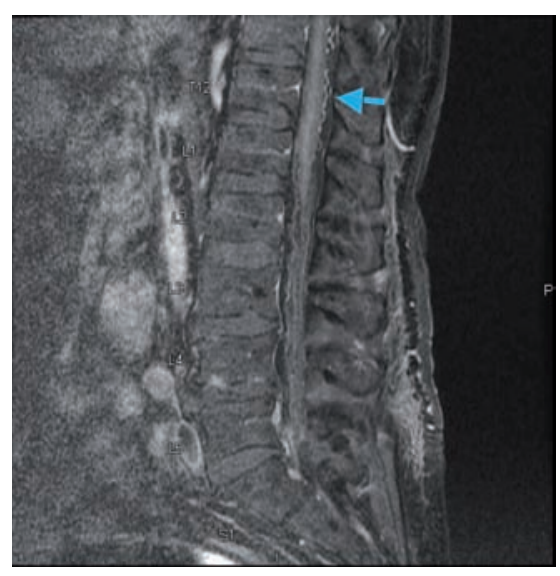

Figure 3. Sagittal T1 FAT saturated sequence post-contrast shows prominent tortuous intradural veins from level T1112 down to the cauda equina nerve roots (arrow)

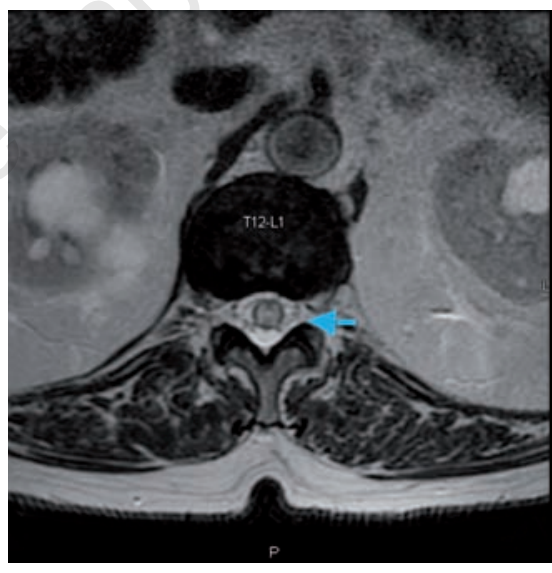

Figure 6. Axial T2 sequence shows hyper intense T2 signal in the central aspect of the lumbar spinal cord with several prominent intradural flow voids (arrow).

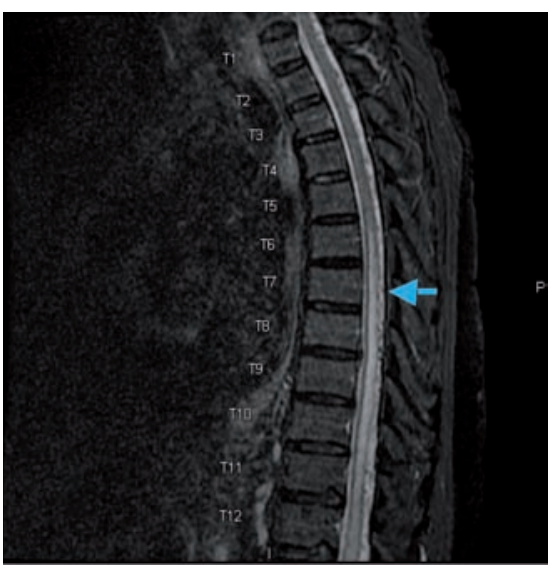

Figure 9. STIR image of the thoracic spine demonstrate hyper intense T2 signal abnormality within the central area of the thoracic spinal cord extending from T6 to the conus medullaris (arrow). 
can be helpful in determining the level of the fistula and can be used for treatment follow-up. ${ }^{5}$ Spinal angiography is the gold standard imaging modality for the diagnosis of SDAVFs. ${ }^{1,6}$ It allows for more detailed characterization of the anatomy of the fistula and its feeding vessels. Differential diagnoses of spinal SDAVFs include demyelinating condition, infarction, inflammation or tumor.

Microsurgical techniques and endovascular embolization represent some of the available treatment options. $1,3,5-9$ There are no exact recommendations and at this time optimal treatment is planned on an individual basis. Surgery can be performed on patients for whom embolization has failed. ${ }^{1,6}$ Endovascular embolization can sometimes lead to incomplete obliteration of the fistula and has been reported to have higher failure rates when compared to surgery. ${ }^{6}$ The goal of treatment is to prevent neurological complications resulting from increased venous congestion. Lower treatment failure rates have been reported with surgery when compared to embolization. ${ }^{6}$

\section{Conclusions}

SDAVF is an important treatable condition that should be considered in the differential diagnosis of patients with progressive myelopathy. Due to the rarity of the condition and its nonspecific clinical presentation, a high index of suspicion is needed for a timely diagnosis. In the absence of awareness, the condition can be often misdiagnosed as a spinal cord tumor leading to surgical exploration. The etiology of SDAVF remains unknown. The gold standard imaging modality is arteriography. Although no concrete guidelines exist for the treatment of spinal SDAVFs, treatment options include surgery and embolization.

\section{References}

1. Kikuchi Y, Miyasaka K. Treatment strategy of spinal arteriovenous malformations based on a simple classification. J Clin Neurosci 1998;5Suppl1:16-9.

2. Borden JA, Wu JK, Shucart WA. A proposed classification for spinal and cranial dural arteriovenous fistulous malformations and implications for treatment. J Neurosurg 1995;82:166-79.

3. Jones BV, Ernst RJ, Tomsick TA, Tew J Jr. Spinal dural arteriovenous fistulas: recognizing the spectrum of magnetic reso- nance imaging findings. J Spinal Cord Med 1997;20:43-8.

4. Rosenblum B, Oldfield EH, Doppman JL, Di Chiro G. Spinal arteriovenous malformations: a comparison of dural arteriovenous fistulas and intradural AVM's in 81 patients. J Neurosurg 1987;67:795-802.

5. Bowen BC, Fraser K, Kochan JP, et al. Spinal dural arteriovenous fistulas: evaluation with MR angiography. AJNR Am J Neuroradiol 1995;16:2029-43.

6. Hessler C, Regelsberger J, Grzyska U, et al. Therapeutic clues in spinal dural arteriovenous fistulas - a 30 year experience of 156 cases. Cent Eur Neurosurg 2010; 71:8-12.

7. Jellema K, Tijssen CC, van Gijn J. Spinal dural arteriovenous fistulas: a congestive myelopathy that initially mimics a peripheral nerve disorder. Brain 2006;129:315064.

8. Klopper HB, Surdell DL, Thorell WE. Type I spinal dural arteriovenous fistulas: historical review and illustrative case. Neuro-surg Focus 2009;26:E3.

9. Ropper AE, Gross BA, Du R. Surgical treatment of Type I spinal dural arteriovenous fistulas. Neurosurg Focus 2012;32: E3. 